\title{
On disjoint crossing families in geometric graphs
}

\author{
Radoslav Fulek*广 Andrew Suk**
}

November 10, 2018

\begin{abstract}
A geometric graph is a graph drawn in the plane with vertices represented by points and edges as straight-line segments. A geometric graph contains a $(k, l)$-crossing family if there is a pair of edge subsets $E_{1}, E_{2}$ such that $\left|E_{1}\right|=k$ and $\left|E_{2}\right|=l$, the edges in $E_{1}$ are pairwise crossing, the edges in $E_{2}$ are pairwise crossing, and every edges in $E_{1}$ is disjoint to every edge in $E_{2}$. We conjecture that for any fixed $k, l$, every $n$-vertex geometric graph with no $(k, l)$-crossing family has at most $c_{k, l} n$ edges, where $c_{k, l}$ is a constant that depends only on $k$ and $l$. In this note, we show that every $n$-vertex geometric graph with no $(k, k)$-crossing family has at most $c_{k} n \log n$ edges, where $c_{k}$ is a constant that depends only on $k$, by proving a more general result which relates extremal function of a geometric graph $F$ with extremal function of two completely disjoint copies of $F$. We also settle the conjecture for geometric graphs with no $(2,1)$-crossing family. As a direct application, this implies that for any circle graph $F$ on 3 vertices, every $n$-vertex geometric graph that does not contain a matching whose intersection graph is $F$ has at most $O(n)$ edges.
\end{abstract}

\section{Introduction}

A topological graph is a graph drawn in the plane with points as vertices and edges as non-selfintersecting arcs connecting its vertices. The arcs are allowed to intersect, but they may not pass through vertices except for their endpoints. Furthermore, the edges are not allowed to have tangencies, i.e., if two edges share an interior point, then they must properly cross at that point. We only consider graphs without parallel edges or self-loops. A topological graph is simple if every pair of its edges intersect at most once. If the edges are drawn as straight-line segments, then the graph is geometric. Two edges of a topological graph cross if their interiors share a point, and are disjoint if they do not have a point in common (including their endpoints).

It follows from Euler's Polyhedral Formula that every simple topological graph on $n$ vertices and no crossing edges has at most $3 n-6$ edges. It is also known that every simple topological graph on $n$ vertices with no pair of disjoint edges has at most $O(n)$ edges [10, [7]. Finding the maximum number of edges in a topological (and geometric) graph with a forbidden substructure has been a classic problem in extremal topological graph theory (see [1, [2, [15], 6], 20], [14], [19], [18], [21]). Many of these problems ask for the maximum number of edges in a topological (or

\footnotetext{
${ }^{*}$ The authors gratefully acknowledge support from the Swiss National Science Foundation Grant No. 200021-

${ }^{\dagger}$ EPFL, Lausanne. Email: radoslav.fulek@epfl.ch

${ }^{\ddagger}$ Courant Institute, New York and EPFL, Lausanne. Email: suk@cims.nyu.edu
} $125287 / 1$ 
geometric) graph whose edge set does not contain a matching that defines a particular intersection graph. Recall that the intersection graph of objects $\mathcal{C}$ in the plane is a graph with vertex set $\mathcal{C}$, and two vertices are adjacent if their corresponding objects intersect. Much research has been devoted to understanding the clique and independence number of intersection graphs due to their applications in VLSI design [8], map labeling [3], and elsewhere.

Recently, Ackerman et al. [4] defined a natural $(k, l)$-grid to be a set of $k$ pairwise disjoint edges that all cross another set of $l$ pairwise disjoint edges. They conjectured

Conjecture 1.1. Given fixed constants $k, l \geq 1$ there exists another constant $c_{k, l}$, such that any geometric graph on $n$ vertices with no natural $(k, l)$-grid has at most $c_{k, l}$ n edges.

They were able to show,

Theorem 1.2. 4] For fixed $k$, an n-vertex geometric graph with no natural $(k, k)$-grid has at most $O\left(n \log ^{2} n\right)$ edges.

Theorem 1.3. 4] An n-vertex geometric graph with no natural $(2,1)$-grid has at most $O(n)$ edges.

Theorem 1.4. 4] An n-vertex simple topological graph with no natural $(k, k)$-grid has at most $O\left(n \log ^{4 k-6} n\right)$ edges.

As a dual version of the natural $(k, l)$-grid, we define a $(k, l)$-crossing family to be a pair of edge subsets $E_{1}, E_{2}$ such that

1. $\left|E_{1}\right|=k$ and $\left|E_{2}\right|=l$,

2. the edges in $E_{1}$ are pairwise crossing,

3. the edges in $E_{2}$ are pairwise crossing,

4. every edge in $E_{1}$ is disjoint to every edge in $E_{2}$.

We conjecture

Conjecture 1.5. Given fixed constants $k, l \geq 1$ there exists another constant $c_{k, l}$, such that any geometric graph on $n$ vertices with no $(k, l)$-crossing family has at most $c_{k, l} n$ edges.

It is not even known if all $n$-vertex geometric graphs with no $k$ pairwise crossing edges has $O(n)$ edges. The best known bound is due to Valtr [22], who showed that this is at most $O(n \log n)$ for every fixed $k$. We extend this result to $(k, k)$-crossing families by proving the following theorem.

Theorem 1.6. An $n$-vertex geometric graph with no $(k, k)$-crossing family has at most $c_{k} n \log n$ edges, where $c_{k}$ is a constant that depends only on $k$.

Let $F$ denote a geometric graph. We say that a geometric graph $G$ contains $F$ as a geometric subgraph if $G$ contains a subgraph $F^{\prime}$ isomorphic to $F$ such that two edges in $F^{\prime}$ cross if and only if the two corresponding edges cross in $F$.

We define $e x(F, n)$ to be the extremal function of $F$, i.e. the maximum number of edges a geometric graph on $n$ vertices can have without containing $F$ as a geometric subgraph. Similarly, we define $e x_{L}(F, n)$ to be the extremal function of $F$, if we restrict ourselves to the geometric graphs all of whose edges can be hit by one line.

Let $F_{2}$ denote a geometric graph, which consists of two completely disjoint copies of a geometric graph $F$. We prove Theorem 1.6 by a straightforward application of the following result. 


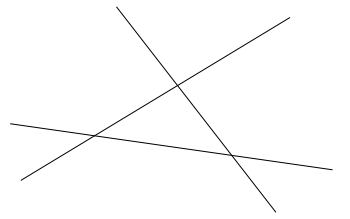

(a) 3 pairwise crossing.

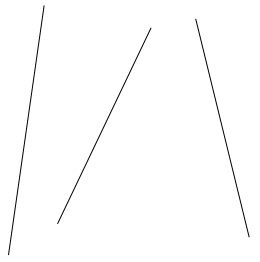

(b) 3 pairwise disjoint.

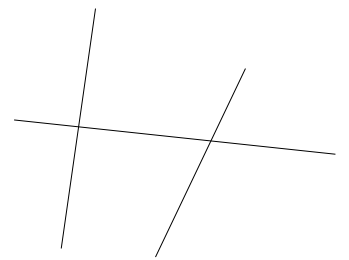

(c) $(2,1)$-grid.

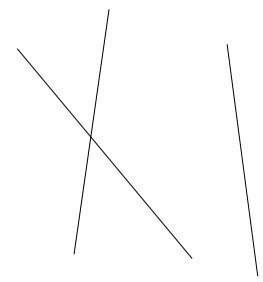

(d) $(2,1)$-crossing family.

Figure 1: Triples of segments corresponding to all circle graphs on three vertices.

Theorem 1.7. $\operatorname{ex}\left(F_{2}, n\right)=O\left(\left(e x_{L}(F, 2 n)+n\right) \log n+e x(F, n)\right)$

Furthermore, we settle Conjecture 1.5 in the first nontrivial case.

Theorem 1.8. An n-vertex geometric graph with no $(2,1)$-crossing family has at most $O(n)$ edges.

Note that Conjecture 1.5 is not true for topological graphs since Pach and Tóth [16] showed that the complete graph can be drawn such that every pair of edges intersect once or twice.

Recall that $F$ is a circle graph if $F$ can be represented as the intersection graph of chords on a circle. By combining Theorem 1.8 with results from [2], 44, and [20], we have the following.

Corollary 1.9. For any circle graph $F$ on 3 vertices, every n-vertex geometric graph that does not contain a matching whose intersection graph is $F$ contains at most $O(n)$ edges.

See Figure 1. We also conjecture the following.

Conjecture 1.10. For any circle graph $F$ on $k$ vertices, there exists a constant $c_{k}$ such that every $n$-vertex geometric graph that does not contain a matching whose intersection graph is $F$, contains at most $c_{k} n$ edges.

As pointed out by Klazar and Marcus [9], it is not hard to modify the proof of the Marcus-Tardos Theorem [19] to show that Conjecture 1.10 is true when the vertices are in convex position.

For simple topological graphs, we have the following

Theorem 1.11. An n-vertex simple topological graph with no $(k, 1)$-crossing family has at most $n(\log n)^{O(\log k)}$ edges.

The paper is organized as follows. Section 2 is devoted to the proof of Theorem 1.7. In Section 3 we establish Theorem 1.8. Finally, the result of Theorem 1.11 about topological graphs is proved in Section 4.

\section{Relating extremal functions}

First, we prove a variant of Theorem 1.7 when all of the edges in our geometric graph can be hit by a line. As in the introduction let $F_{2}$ denote a geometric graph, which consists of two completely disjoint copies of a geometric graph $F$. We will now show that the extremal function $e x_{L}\left(F_{2}, n\right)$ is not far from $e x_{L}(F, n)$. 
Theorem 2.1. $e x_{L}\left(F_{2}, n\right) \leq O\left(\left(n+e x_{L}(F, 2 n)\right) \log n\right)$.

Proof. Let $G$ denote a geometric graph on $n$ vertices that does not contain $F_{2}$ as a geometric subgraph, and all the edges of $G$ can be hit by a line. By a standard perturbation argument we can assume that the vertices of $G$ are in general position. As in [5], a halving edge uv is a pair of the vertices in $G$ such that the number of vertices on each side of the line through $u$ and $v$ is the same.

Lemma 2.2. There exists a directed line $\vec{l}$ such that the number of edges in $G$ that lies completely to the left or right of $\vec{l}$ is at most $2 e x_{L}(F, n / 2)+5 n$.

Proof. If $n$ is odd we can discard one vertex of $G$, thereby loosing at most $n$ edges. Therefore we can assume $n$ is even, and it suffices to show that there exists a directed line $\vec{l}$ such that the number of edges in $G$ that lies completely to the left or right of $\vec{l}$ is at most $2 e x_{L}(F, n / 2)+4 n$.

Let $u v$ be a halving edge, and let $\vec{l}$ denote the directed line containing vertices $u$ and $v$ with direction from $u$ to $v$. Let $e(\vec{l}, L)$ and $e(\vec{l}, R)$ denote the number of edges on the left and right side of $\vec{l}$ respectively. Without loss of generality, we can assume that $e(\vec{l}, L) \leq e(\vec{l}, R)$. We will rotate $\vec{l}$ such that it remains a halving line at the end of each step, until it reaches a position where the number of edges on both sides of $\vec{l}$ is roughly the same.

We start by rotating $\vec{l}$ counterclockwise around $u$ until it meets the next vertex $w$ of $G$. If initially $w$ lies to the right of $\vec{l}$, then in the next step we will rotate $\vec{l}$ around $u$ (again). See Figure $2(\mathrm{a})$. Otherwise if $w$ was on the left side of $\vec{l}$, then in the next step we will rotate $\vec{l}$ around vertex $w$. See Figure 2(b), Clearly after each step in the rotation, there are exactly $n / 2$ vertices on each side of $\vec{l}$.

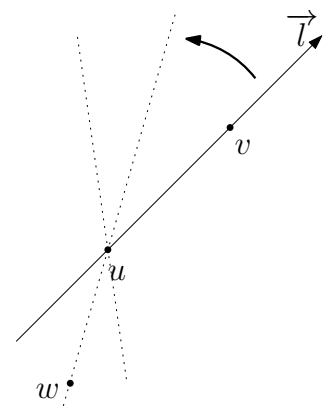

(a) $w$ lies to the right of $\vec{l}$.

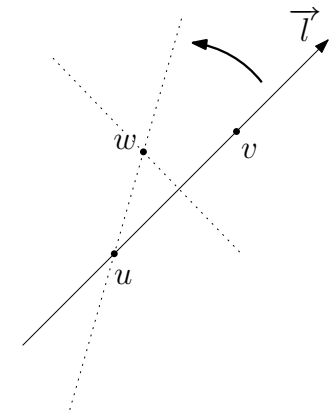

(b) $w$ lies to the left of $\vec{l}$.

Figure 2: Halving the vertices of $G$

After several rotations, $\vec{l}$ will eventually contain points $u$ and $v$ again, with direction from $v$ to $u$. At this point we have $e(\vec{l}, L) \geq e(\vec{l}, R)$. Since the number of edges on the right side (and left side) changes by at most $n$ after each step in the rotation, at some point in the rotation we must have

$$
|e(\vec{l}, L)-e(\vec{l}, R)| \leq 2 n .
$$

Since $G$ does not contain a $F_{2}$ as a geometric subgraph, this implies that 


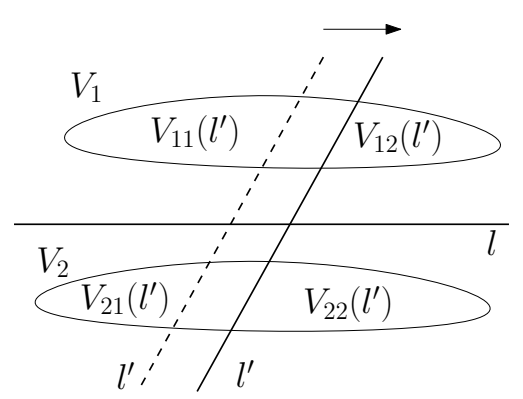

Figure 3: The final partition of the vertex set of $G$

$$
e(\vec{l}, L) \leq e x_{L}(F, n / 2)+2 n
$$

and

$$
e(\vec{l}, R) \leq e x_{L}(F, n / 2)+2 n .
$$

Therefore for any $n$, there exists a directed line $\vec{l}$ such that the number of edges in $G$ that lies completely to the left or right of $\vec{l}$ is at most $2 e x_{L}(F, n / 2)+5 n$.

By Lemma 2.2 we obtain a line $l$, which partition the vertices of $G$ into two equal (or almost equal if $n$ is odd) sets $V_{1}$ and $V_{2}$. Let $E^{\prime}$ denote the set of edges between $V_{1}$ and $V_{2}$. By the Ham-Sandwich Cut Theorem [11, there exists a line $l^{\prime}$ that simultaneously bisects $V_{1}$ and $V_{2}$. Let $V_{11}\left(l^{\prime}\right)$ and $V_{12}\left(l^{\prime}\right)$ denote the resulting parts of $V_{1}$, and let $V_{21}\left(l^{\prime}\right)$ and $V_{22}\left(l^{\prime}\right)$ denote the resulting parts of $V_{2}$.

Observe that we can translate $l^{\prime}$ along $l$ into a position where the number of edges in $E^{\prime}$ that lie completely to the left and completely to the right of $l^{\prime}$ is roughly the same. In particular, we can translate $l^{\prime}$ along $l$ such that the number of edges in $E^{\prime}$ that lies completely to its left or right side is at most $e x_{L}(F, n)+e x_{L}(F, n / 2+1)+n$ (see Figure 2). Indeed, assume that the number of edges in $E^{\prime}$ between, say, $V_{12}\left(l^{\prime}\right)$ and $V_{22}\left(l^{\prime}\right)$ is more than $e x_{L}(F, n / 2+1)$. As we translate $l^{\prime}$ to the right, the number of edges that lie completely to the right of $l^{\prime}$ changes by at most $n$ as $l^{\prime}$ crosses a single vertex in $G$. Therefore we can translate $l^{\prime}$ into the leftmost position where the number of edges in $E^{\prime}$ between $V_{12}\left(l^{\prime}\right)$ and $V_{22}\left(l^{\prime}\right)$ drops below $e x_{L}(F, n / 2+1)+n+1$. Moreover, at this position the number of edges in $E^{\prime}$ between $V_{11}\left(l^{\prime}\right)$ and $V_{21}\left(l^{\prime}\right)$ still cannot be more than $\operatorname{ex}_{L}(F, n)$ since $G$ does not contain $F_{2}$ as a geometric subgraph.

Thus, all but at most $3 e x_{L}(F, n / 2+1)+e x_{L}(F, n)+6 n$ edges of $G$ are the edges between $V_{11}\left(l^{\prime}\right)$ and $V_{22}\left(l^{\prime}\right)$, and between $V_{12}\left(l^{\prime}\right)$ and $V_{21}\left(l^{\prime}\right)$. Notice that there exists $k,-1 / 4 \leq k \leq 1 / 4$, such that $\left|V_{11}\left(l^{\prime}\right)\right|+\left|V_{22}\left(l^{\prime}\right)\right|=n(1 / 2+k)$, and $\left|V_{12}\left(l^{\prime}\right)\right|+\left|V_{21}\left(l^{\prime}\right)\right|=n(1 / 2-k)$. Finally, we are in the position to state the recurrence, whose closed form gives the statement of the theorem.

$$
e x_{L}\left(F_{2}, n\right) \leq e x_{L}\left(F_{2}, n(1 / 2+k)\right)+e x_{L}\left(F_{2}, n(1 / 2-k)\right)+3 e x_{L}(F, n / 2+1)+e x_{L}(F, n)+6 n .
$$


By a routine calculation which is indicated below, we have

$$
\begin{aligned}
e x_{L}\left(F_{2}, n\right) \leq & \log _{\frac{4}{3}}\left(n\left(\frac{1}{2}+k\right)\right)\left(6 n\left(\frac{1}{2}+k\right)+4 e x_{L}\left(F_{2}, 2 n\left(\frac{1}{2}+k\right)\right)\right)+ \\
& \log _{\frac{4}{3}}\left(n\left(\frac{1}{2}-k\right)\right)\left(6 n\left(\frac{1}{2}-k\right)+4 e x_{L}\left(F_{2}, 2 n\left(\frac{1}{2}-k\right)\right)\right)+ \\
& 4 \operatorname{ex}_{L}(F, n)+6 n \\
\leq & \log _{\frac{4}{3}} n\left(4 e x_{L}(F, 2 n)+6 n\right)
\end{aligned}
$$

Finally, we show how Theorem 2.1 implies Theorem 1.7 .

Proof of Theorem 1.7. Let $G=(V, E)$ denote the geometric graph not containing $F_{2}$ as a subgraph. Similarly, as in the proof of Lemma 2.2 we can find a halving line $l$ that hits all but $2 e x(F, n / 2)+5 n$ edges of $G$. Now, the claim follows by using Theorem 2.1.

Theorem 1.6 follows easily by using Theorem 1.7 with a result from [22, which states that every $n$-vertex geometric graph whose edges can be all hit by a line and does not contain $k$ pairwise crossing edges has at most $O(n)$ edges and at most $O(n \log n)$ edges if we do not require a single line to hit all the edges.

\section{Geometric graphs with no $(2,1)$-crossing family}

In this section we will prove Theorem 1.8 . Our main tool is the following theorem by Tóth and Valtr

Theorem 3.1. 21] Let $G=(V, E)$ be an n-vertex geometric graph. If $G$ does not contain a matching consisting of 5 pairwise disjoint edges, then $|E(G)| \leq 64 n+64$.

Theorem 1.8 immediately follows from the following theorem.

Theorem 3.2. Every $n$-vertex geometric graph with no $(2,1)$-crossing family has at most $64 n+64$ edges.

Proof. For sake of contradiction, let $G=(V, E)$ be a vertex-minimal counter example, i.e. $G$ is a geometric graph on $n$ vertices which has more than $64 n+64$ edges and $G$ does not contain a (2,1)-crossing family. Hence every vertex in $G$ has degree at least 65 . Let $M$ denote the maximum matching in $G$ consisting of pairwise disjoint edges and let $V_{M}$ denote the vertices in $M$. Since $|E(G)|>64 n+64$, Theorem 3.1 implies that $|M| \geq 5$. We say that two edges intersect if they cross or share an endpoint. The following simple observation is crucial in the subsequent analysis.

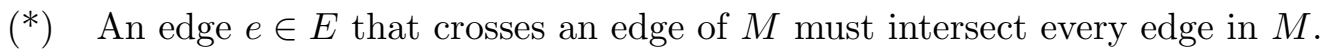

Indeed, otherwise we would obtain a $(2,1)$-crossing family. We call an endpoint $v$ of an edge in $M$ good if every ray starting at $v$ misses at least one edge in $M$. See Figure 4(b). 
Lemma 3.3. For $|M| \geq 4$, at least $|M|-2$ of the endpoints in $M$ are good.

Proof. We proceed by induction on $|M|$. Assume $|M|=4$. If every triple in $M$ has a good vertex, then clearly we have at least two good vertices. Otherwise the only matching consisting of three pairwise disjoint edges with no good vertices is the one in Figure 4(a), By a simple case analysis, adding a disjoint edge to this matching creates two good vertices (see Appendix A). For the inductive step when $|M|>4$, we choose an arbitrary 4-tuple of edges in the matching. By the above discussion, the 4-tuple has at least one good endpoint. By removing the edge incident to this good vertex, the statement follows by the induction hypothesis.

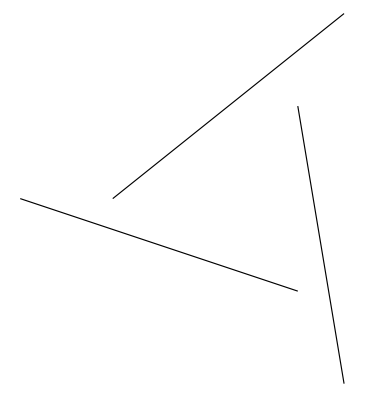

(a)

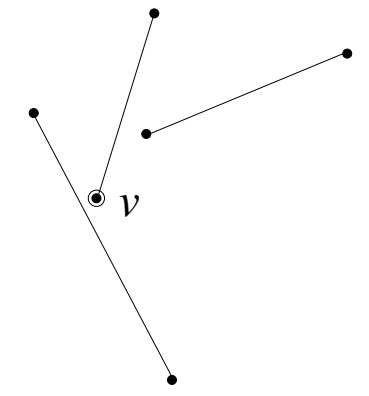

(b)

Figure 4: (a) Special case in Lemma 3.3, (b) Matching $M$ of size 3 with one good vertex $v$

Thus by $\left({ }^{*}\right)$, a good endpoint cannot be incident to an edge that crosses any of the edges in $M$. Let

1. $V_{g} \subseteq V_{M}$ denote the set of good endpoints in $M$.

2. $V_{1} \subset V \backslash V_{M}$ be the subset of the vertices such that for $v \in V_{1}$, every edge incident to $v$ does not cross any of the edges in $M$,

3. and $V_{2}=V \backslash\left(V_{M} \cup V_{1}\right)$. Hence for $v \in V_{2}$, there exists an edge incident to $v$ that intersects every edge in $M$.

See Figure 5(a). By Lemma 3.3, $\left|V_{g}\right| \geq|M|-2=\left|V_{M}\right| / 2-2$. By maximality of $M$, there are no edges between $V_{1}$ and $V_{2}$ and $V_{1}$ is an independent set. Now notice the following observation.

Observation 3.4. There exists a good vertex in $V_{g}$ that has at least three neighbors in $V_{2}$.

Proof. For sake of contradiction, suppose that each vertex in $V_{g}$ has at most two neighbors in $V_{2}$. Then let $G^{\prime}=\left(V^{\prime}, E^{\prime}\right)$ denote a subgraph of $G$ such that $V^{\prime}=V_{M} \cup V_{1}$ and $E^{\prime}$ consists of the edges that do not cross any of the edges of $M$ and whose endpoints are in $V_{1} \cup V_{M}$. Since $|M| \geq 5$, $G^{\prime}$ must be a planar graph since otherwise we would have a $(2,1)$-crossing family. Therefore $E^{\prime} \leq 3\left(\left|V_{1}\right|+\left|V_{M}\right|\right)$. 
On the other hand, by minimality of $G$, each vertex in $V_{1}$ has degree at least 65 in $G^{\prime}$, and each vertex in $V_{g}$ has degree at least 63 in $G^{\prime}$. Therefore by applying Lemma 3.3 , we have

$$
\frac{1}{2}\left(65\left|V_{1}\right|+63\left(\frac{\left|V_{M}\right|}{2}-2\right)\right) \leq\left|E^{\prime}\right| \leq 3\left|V_{1}\right|+3\left|V_{M}\right| .
$$

This implies

$$
59\left|V_{1}\right|+25\left|V_{M}\right| \leq 126
$$

which is a contradiction since $\left|V_{M}\right| \geq 10(|M| \geq 5)$.

Let $v \in V_{g}$ be a good vertex such that $v$ has at least 3 neighbors in $V_{2}$. Let $e=v v_{1}, f=v v_{2}$, $g=v v_{3}$, and $m$ be edges in $G$ such that $v_{1}, v_{2}, v_{3} \in V_{2}, m \in M$, and $v$ is a good vertex incident to $m$. Furthermore, we will assume that $g, e, m, f$ appear in clockwise order around $v$. By $\left(^{*}\right)$ there is an edge $e^{\prime}$ incident to $v_{1}$ having non-empty intersection with every edge in $M$. Similarly, we can find such an edge $f^{\prime}$ incident to $v_{2}$ (possibly $f^{\prime}=e^{\prime}$ ). The edges $e^{\prime}$ and $f^{\prime}$ must have non-empty intersection with $f$ and $e$, respectively (see Figure 5(b) . Otherwise we would obtain a (2,1)-crossing family in $G$ consisting of $e, f^{\prime}$ and an edge from $M$, or $e^{\prime}, f$ and an edge from $M$.

However, a third edge $g$ cannot have a non-empty intersection with both $e^{\prime}$ and $f^{\prime}$. Hence, we obtain a (2,1)-crossing family in $G$ consisting of $g, e^{\prime}$ and an edge from $M$, or $g, f^{\prime}$ and an edge from $M$. Thus, there is no minimal counter example and that concludes the proof.

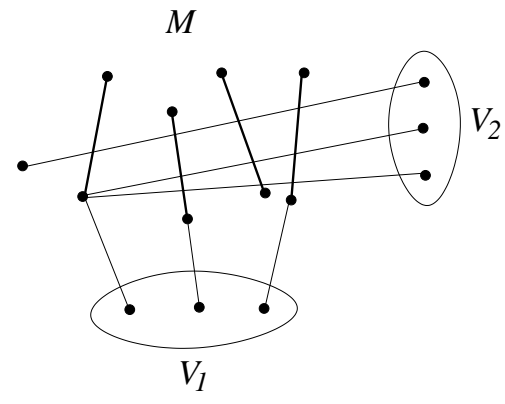

(a)

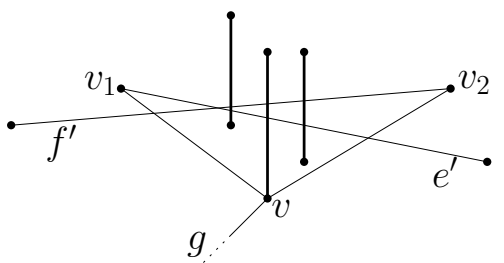

(b)

Figure 5: (a) $M, V_{1}$, and $V_{2}$, (b) Situation around the vertex $v$

We note that by a more tedious case analysis, one could improve the upper bound in Theorem 3.2 to $15 n$.

\section{Simple topological graphs with no $(k, 1)$-crossing family}

In this section, we will prove Theorem 1.11 which will require the following two lemmas. The first one is due to Fox and Pach.

Lemma 4.1. 6] Every n-vertex simple topological graph with no $k$ pairwise crossing edges has at most $n(\log n)^{c_{1} \log k}$ edges, where $c_{1}$ is an absolute constant. 
As defined in [17, the odd-crossing number odd-cr $(G)$ of a graph $G$ is the minimum possible number of unordered pairs of edges that crosses an odd number of times over all drawings of $G$. The bisection width of a graph $G$, denoted by $b(G)$, is the smallest nonnegative integer such that there is a partition of the vertex set $V=V_{1} \dot{\cup} V_{2}$ with $\frac{1}{3} \cdot|V| \leq V_{i} \leq \frac{2}{3} \cdot|V|$ for $i=1,2$, and $\left|E\left(V_{1}, V_{2}\right)\right|=b(G)$. The second lemma required is due to Pach and Tóth, which relates the odd-crossing number of a graph to its bisection width.

Lemma 4.2. [16] There is an absolute constant $c_{2}$ such that if $G$ is a graph with $n$ vertices of degrees $d_{1}, \ldots, d_{n}$, then

$$
b(G) \leq c_{2} \log n \sqrt{\operatorname{odd}-\operatorname{cr}(G)+\sum_{i=1}^{n} d_{i}^{2}} .
$$

Since all graphs have a bipartite subgraph with at least half of its edges, Theorem 1.6 immediately follows from the following Theorem.

Theorem 4.3. Every $n$ vertex simple topological bipartite graph with no $(k, 1)$-crossing family has at most $c_{3} n \log ^{c_{4} \log k} n$ edges, where $c_{3}, c_{4}$ are absolute constants.

Proof. We proceed by induction on $n$. The base case is trivial. For the inductive step, the proof falls into two cases.

Case 1. Suppose there are at least $|E(G)|^{2} /\left(\left(2 c_{2}\right)^{2} \log ^{6} n\right)$ disjoint pair of edges in $G$. Then by defining $D(e)$ to be the set of edges disjoint from edge $e$, we have

$$
\frac{2|E(G)|}{\left(2 c_{2}\right)^{2} \log ^{6} n} \leq \frac{\sum_{e \in E(G)}|D(e)|}{|E(G)|}
$$

Hence there exists an edge that is disjoint to at least $2|E(G)| /\left(\left(2 c_{2}\right)^{2} \log ^{6} n\right)$ other edges. By Lemma 4.1 we have

$$
\frac{2|E(G)|}{\left(2 c_{2}\right)^{2} \log ^{6} n} \leq n(\log n)^{c_{1} \log k},
$$

which implies $|E(G)| \leq c_{3} n \log ^{c_{4} \log k} n$ for sufficiently large constants $c_{3}, c_{4}$.

Case 2. Suppose there are at most $|E(G)|^{2} /\left(\left(2 c_{2}\right)^{2} \log ^{6} n\right)$ disjoint pair of edges in $G$. Since $G$ is bipartite, let $V_{a}$ and $V_{b}$ be its vertex class. By applying a suitable homeomorphism to the plane, we can redraw $G$ such that

1. the vertices in $V_{a}$ are above the line $y=1$, the vertices in $V_{b}$ are below the line $y=0$,

2. edges in the strip $0 \leq y \leq 1$ are vertical segments,

3. we have not created nor removed any crossings.

Now we reflect the part of $G$ that lies above the $y=1$ line about the $y$-axis. Then erase the edges in the strip $0 \leq y \leq 1$ and replace them by straight line segments that reconnects the corresponding 

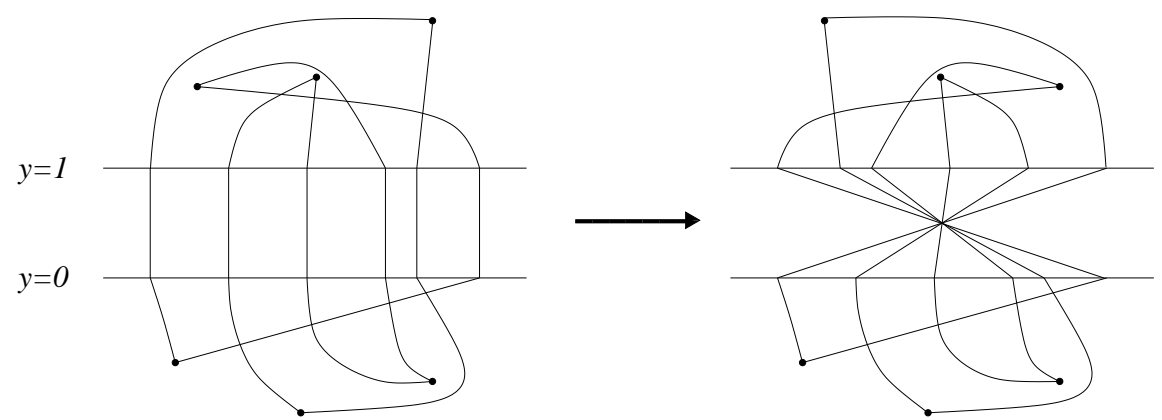

Figure 6: Redrawing procedure

pairs on the line $y=0$ and $y=1$. See Figure 4 , and note that our graph is no longer simple. Since there are at most $\sum_{v \in V(G)} d^{2}(v) \leq 2|E(G)| n$ pair of edges that share a vertex in $G$, this implies

$$
\text { odd-cr }(G) \leq \frac{|E(G)|^{2}}{\left(2 c_{2}\right)^{2} \log ^{6} n}+2|E(G)| n .
$$

By Lemma 4.2 , there is a partition of the vertex set $V=V_{1} \dot{\cup} V_{2}$ with $\frac{1}{3} \cdot|V| \leq V_{i} \leq \frac{2}{3} \cdot|V|$ for $i=1,2$ and

$$
b(G) \leq c_{2} \log n \sqrt{\frac{|E(G)|^{2}}{\left(2 c_{2}\right)^{2} \log ^{6} n}+4 n|E(G)|} .
$$

If

$$
\frac{|E(G)|^{2}}{\left(2 c_{2}\right)^{2} \log ^{6} n} \leq 4 n|E(G)|
$$

then we have $|E(G)| \leq c_{3} n \log ^{c_{4} \log k} n$ and we are done. Therefore we can assume

$$
b(G) \leq c_{2} \log n \sqrt{\frac{2|E(G)|^{2}}{\left(2 c_{2}\right)^{2} \log ^{6} n}} \leq \frac{|E(G)|}{\log ^{2} n} .
$$

Let $\left|V_{1}\right|=n_{1}$ and $\left|V_{2}\right|=n_{2}$. By the induction hypothesis we have

$$
\begin{aligned}
|E(G)| & \leq b(G)+c_{3} n_{1} \log ^{c_{4} \log k} n_{1}+c_{3} n_{2} \log ^{c_{4} \log k} n_{2} \\
& \leq \frac{|E(G)|}{\log ^{2} n}+c_{3} n \log ^{c_{4} \log k}(2 n / 3) \\
& \leq \frac{|E(G)|}{\log ^{2} n}+c_{3} n(\log n-\log (3 / 2))^{c_{4} \log k},
\end{aligned}
$$

which implies

$$
|E(G)| \leq c_{3} n \log ^{c_{4} \log k} n \frac{(1-\log (3 / 2) / \log n)^{c_{4} \log k}}{1-1 / \log ^{2} n} \leq c_{3} n \log ^{c_{4} \log k} n .
$$


For small values of $k$, one can obtain better bounds by replacing Lemma 4.1 with a Theorem of Pach et. al. [14] and Ackerman [1] to obtain

Theorem 4.4. For $k>4$, every $n$ vertex simple topological graph with no $(k, 1)$-crossing family has at most $O\left(n \log ^{2 k+2} n\right)$ edges. For $k=2,3,4$, every $n$ vertex simple topological graph with no $(k, 1)$-crossing family has at most $O\left(n \log ^{6} n\right)$ edges.

\section{Appendix}

\section{A Four disjoint edges}

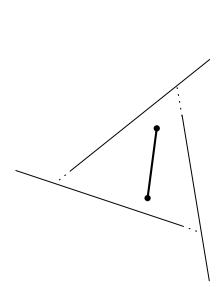

(a)

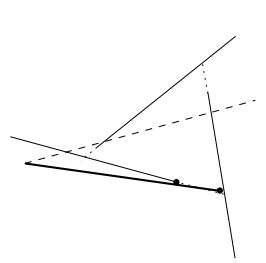

(b)

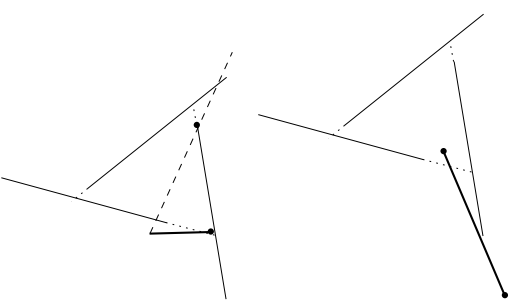

(c)

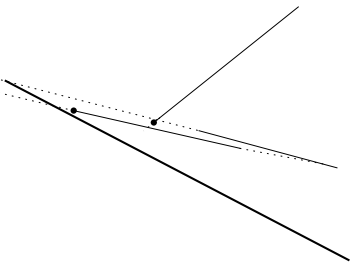

(e)

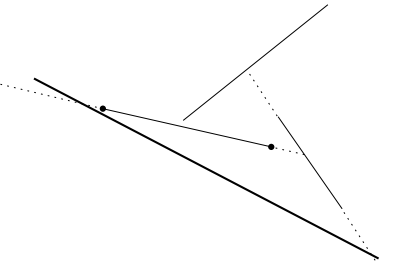

(f) (d)

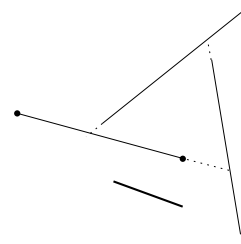

$(\mathrm{g})$

Figure 7: Possible configuration of 4 pairwise disjoint edges, the additional edge is bold, and good vertices are marked by small discs

Suppose that we have three pairwise disjoint edges in the plane, whose combinatorial configuration is that of the configuration in Figure 4(a). Let $T$ denote the triangle we get by prolonging the edges until they hit another edge. In what follows we show that by adding additional edge to this configuration, so that all the edges remain pairwise disjoint, we obtain at least two good endpoints (as defined in the proof of Theorem 1.8).

There are three cases to check:

1. The additional edge is completely inside of the triangle $T$ (see Figure 7(a)). The two good points are the endpoints of the additional edge.

2. The additional edge $e$ has one endpoint in the inside the triangle $T$ and the one outside of it. Clearly, the endpoint of $e$ inside of $T$ is a good endpoint. If the other endpoint of $e$ is not good, we can easily see (see Figure $7(\mathrm{~b}), 7(\mathrm{c})$ and $7(\mathrm{~d}))$, that one of the remaining endpoints is good. 
3. The additional edge is completely outside of the triangle $T$. There are three cases to be checked (see Figure 7(e), 7(f) and 7(g) , according to where the lines through our segments meet.

\section{References}

[1] E. Ackerman, 2006. On the maximum number of edges in topological graphs with no four pairwise crossing edges. In Proceedings of the Twenty-Second Annual Symposium on Computational Geometry (Sedona, Arizona, USA, June 05 - 07, 2006). SCG '06. ACM, New York, NY, 259-263.

[2] P.K. Agarwal, B. Aronov, J. Pach, R. Pollack, and M. Sharir, Quasi-Planar Graphs Have a Linear Number of Edges. In Proceedings of the Symposium on Graph Drawing (September 20 22, 1995). F. Brandenburg, Ed. Lecture Notes In Computer Science, vol. 1027. Springer-Verlag, London, 1-7.

[3] P.K. Agarwal, M. van Kreveld, and S. Suri, Label placement by maximum independent set in rectangles, Comput. Geom. Theory Appl. 11 (1998), 209-218.

[4] E. Ackerman, J. Fox, J. Pach, and A. Suk, On grids in topological graphs, Proc. 25th ACM Symp. on Computational Geometry (SoCG), University of Aarhus, Denmark, June 2009, 403-412.

[5] T. Dey, Improved Bounds on Planar k-sets and k-levels, Discrete Comput. Geom, 1997, 19, $156-161$

[6] J. Fox and J. Pach, Coloring $K_{k}$-free intersection graphs of geometric objects in the plane. In Proceedings of the Twenty-Fourth Annual Symposium on Computational Geometry (College Park, MD, USA, June 09 - 11, 2008). SCG '08. ACM, New York, NY, 346-354.

[7] R. Fulek and J. Pach, A computational approach to Conway's thrackle conjecture, Computational Geometry: Theory and Application, 2010, to appear.

[8] D. S. Hochbaum and W. Maass, Approximation schemes for covering and packing problems in image processing and VLSI, J. ACM 32 (1985), 130-136.

[9] M. Klazar and A. Marcus,Extensions of the linear bound in the Fredi-Hajnal conjecture, Adv. in Appl. Math. 38 (2006), 258-266.

[10] L. Lovász, J. Pach and M. Szegedy: On Conway's thrackle conjecture, Discrete. Comput. Geom. 18(4) (1997), 369376.

[11] J. Matouśek, Lectures on Discrete Geometry. Springer-Verlag New York, Inc. 2002.

[12] A. Marcus, G. Tardos, Excluded permutation matrices and the Stanley-Wilf conjecture, Journal of Combinatorial Theory Series A, v.107 n.1, p.153-160, July 2004.

[13] J. Pach and J. Solymosi, Crossing patterns of segments. J. Comb. Theory Ser. A 96, 2 (Nov. 2001), 316-325. 
[14] J. Pach, R. Radoicic, G. Toth. Relaxing planarity for topological graphs, J. Akiyama, M. Kano (Eds.): Discrete and Computational Geometry, Japanese Conference, JCDCG 2002, Tokyo, Japan, Lecture Notes in Computer Science, 2866, Springer, 2003, 221-232.

[15] J. Pach, F. Shahrokhi, and M. Szegedy. 1994. Applications of the crossing number. In Proceedings of the Tenth Annual Symposium on Computational Geometry (Stony Brook, New York, United States, June 06 - 08, 1994). SCG '94. ACM, New York, NY, 198-202.

[16] J. Pach and G. Tóth, Disjoint edges in topological graphs,in: Combinatorial Geometry and Graph Theory (J. Akiyama et al., eds.),Lecture Notes in Computer Science 3330,Springer-Verlag, Berlin, 2005, 133-140.

[17] J. Pach and G. Tóth, Which crossing number is it anyway?. J. Comb. Theory Ser. B 80, 2 (Nov. 2000), 225-246.

[18] J. Pach, J. Töröcsik, Some Geometric Applications of Dilworth's Theorem. Discrete \& Computational Geometry 12: 1-7 (1994)

[19] G. Tardos, G. Tóth: Crossing stars in topological graphs, Japan Conference on Discrete and Computational Geometry 2004, Lecture Notes in Computer Science 3742 Springer-Verlag, Berlin, 184-197.

[20] G. Tóth, 2000. Note on geometric graphs. J. Comb. Theory Ser. A 89, 1 (Jan. 2000), 126-132.

[21] G. Tóth and P. Valtr. 1998 Geometric Graphs with Few Disjoint Edges. Technical Report. UMI Order Number: 98-22., Center for Discrete Mathematics \& Theoretical Computer Science.

[22] P. Valtr, Graph drawings with no $k$ pairwise crossing edges, in: G. Di Battista (ed.), Graph Drawing '97, Rome, September 1997, pp. 205-218 